\title{
Lieux de mémoire et de pouvoir chez les Oman-Jagou en pays Idaatcha : Ėgbakokou et Yaka, symboles de la dualité du pouvoir local.
}

\author{
Opêoluwa Blandine Agbaka, \\ INMAAC-Université d'Abomey-Calavi, Bénin.
}

\begin{abstract}
Résumé: En plein cœur de Dassa-Zoumè, chef-lieu du département des Collines au centre nord du Bénin, deux lieux symbolisent la vitalité toujours renouvelée de la mémoire collective d'un royaume dont les origines remonteraient au XVIè siècle (Adjiba 2006). En effet, Les lieux de mémoire sont des creusets d'ancrage de la mémoire, mais d'une mémoire généralement collective. Leur vitalité est souvent l'objet d'un entretien périodique pour que la remémoration qui constitue leur essence vitale ne s'estompe pas. Au sein des communautés béninoises urbaines ou rurales, la célébration de la mémoire communautaire à travers des lieux dédiés à cet effet est un usage assez courant. Le présent article propose toutefois de présenter les cas particuliers d'Ègbakokou et Yaka, deux lieux représentant la dualité du pouvoir au sommet de la royauté en pays Idaatcha. Leur symbolique rime avec mémoire et pouvoir. Ancrés profondément dans l'organisation structurelle du royaume d'Igbo Idaatcha, ces deux lieux constituent chez les Oman-Jagou, deux altérités de la même réalité : l'expression et la manifestation du pouvoir. Si le Jagou (roi) règne sur Igbo Idaatcha et siège à Ègbakokou, l'Obaloké, le chef spirituel et la première autorité religieuse du royaume siège à Yaka.
\end{abstract}

Mots clés : lieux de mémoire ; pouvoir ; Oman-Jagou ; Idaatcha ; Bénin

\begin{abstract}
In the heart of Dassa-Zoumè, the capital of the department "des Collines", in north central Benin, two places symbolize the ever-renewed vitality of the collective memory of a kingdom whose origins date back to the 16th century, Adjiba (2006). Indeed, Places of Memory are crucibles for anchoring memory, but of a generally collective memory. Their vitality is often the subject of periodic maintenance so that the memory which constitutes their vital essence does not fade. In Benin's urban or rural communities, the celebration of community memory through places dedicated to this effect is quite common. This article proposes however to present the very interesting cases of Ègbakokou and Yaka, two places which represented the dual roots of royal power in Idaatcha land. Their symbolism rhymes with memory and power. Rooted deeply in the structural organization of the kingdom of Igbo Idaatcha, these two places constitute among the Oman-Jagou, two other sides of the same reality: the expression and the manifestation of power. If the Jagou (king) reigns over Igbo Idaatcha and sits in Egbakokou, the Obaloké, the spiritual leader and the first religious authority of the kingdom sits in Yaka.
\end{abstract}

Key words : Places of memory ; Power ; Oman-Jagou ; Idaatcha ; Benin

Opêoluwa Blandine AGBAKA est titulaire d'un doctorat en histoire, histoire de l'art et archéologie de l'Université libre de Bruxelles. Elle est enseignante-chercheure à I'Institut National des Métiers d'Art, d'Archéologie et de la Culture (Université d'Abomey-Calavi, Bénin). Email :kaddine2@yahoo.fr

Culture and Local Governance / Culture et gouvernance locale, vol. 7, no. 1-2, 2020. ISSN 1911-7469

Centre on Governance, University of Ottawa, 120 university, Ottawa, Ontario, Canada K1N 6N5 


\section{Introduction}

La reconnaissance de l'immatérialité du patrimoine, au niveau international par I'UNESCO, se concrétise en 1997 avec la mise en place de la Proclamation des chefs-d'œuvre du patrimoine oral et immatériel de l'humanité. L'adoption de la Convention pour la Sauvegarde du patrimoine immatériel en 2003 consacre le processus de reconnaissance de l'immatériel comme une catégorie de patrimoine qui se définit dans l'article 2 de la Convention comme étant :

Les pratiques, représentations, expressions, connaissances et savoir-faire - ainsi que les instruments, objets, artefacts et espaces culturels qui leur sont associés - que les communautés, les groupes et, le cas échéant, les individus reconnaissent comme faisant partie de leur patrimoine culturel. Ce patrimoine culturel immatériel, transmis de génération en génération, est recréé en permanence par les communautés et groupes en fonction de leur milieu, de leur interaction avec la nature et de leur histoire, et leur procure un sentiment d'identité et de continuité, contribuant ainsi à promouvoir le respect de la diversité culturelle et la créativité humaine.

L'une des particularités de la culture béninoise réside en la richesse de son patrimoine immatériel. Les espaces socioculturels, qu'ils soient associés à des pratiques religieuses ou culturelles, représentent des symboles d'expression de la diversité culturelle des communautés béninoises. Les lieux de mémoire occupent une place importante dans les pratiques commémoratives et soutiennent les manifestations de la mémoire collective des différentes communautés. La volonté manifeste de célébrer l'identité collective et de marquer la particularité des ensembles culturels homogènes s'est renforcée au fil des années au Bénin. En témoigne la prolifération des festivals à caractère communautaire dont les plus connus sont : Nonvitcha pour les peuples Xwla, la Gaani pour les Bariba, le Wémèxwé chez les peuples de la Vallée de l'Ouémé, le festival du Danxomè chez les peuples Fon du plateau d'Abomey, le Festival des Arts et Cultures Idaatcha (FACl) pour les communautés Idaatcha, etc. Ces festivals, creusets par excellence d'expression et de manifestation des éléments du patrimoine culturel immatériel $(\mathrm{PCl})$ sont également généralement associés à des lieux qui représentent le support matériel de la mémoire collective.

Cette contribution présente l'importance et la particularité de deux lieux de mémoire : Ègbakokou et Yaka qui symbolisent la dualité de l'exercice du pouvoir en pays idaatcha. En effet, dans les pratiques socioculturelles des Oman-Jagou, le clan majoritaire d'Igbo Idaatcha, le pouvoir royal est structuré en deux grands noyaux: le pouvoir exécutif et le pouvoir religieux. Le royaume d'Igbo Idaatcha ${ }^{1}$ dont les origines remonteraient au XVIè siècle d'après Adjiba (2006) a une population qui s'étend sur les actuelles communes de Dassa-Zoumè et de Glazoué dans le département des Collines au centre nord du pays. Au Bénin, les systèmes traditionnels de gestion de la société cohabitent avec l'organisation administrative moderne. Le royaume d'Igbo Idaatcha, à l'instar de plusieurs autres royaumes du pays a conservé son organisation traditionnelle de gestion, reflétée par Yaka et Ègbakokou.

En dehors de la symbolique mémorielle et royale de ces deux espaces patrimoniaux, se profile la problématique actuelle de conservation et de gestion des lieux de mémoire dans le respect des normes internationales. En effet, la problématique de la conservation de ces lieux pose un sérieux problème au regard des questions d'authenticité. Au final, le réel problème qui se pose lorsque l'on aborde la conservation de ce genre de sites est de définir exactement les acteurs de la légitimation de l'authenticité. Qui légitime l'authenticité d'un lieu de mémoire? Les professionnels ou les communautés ? Authentique pour qui ? Et pourquoi ? 
La description de ces lieux et des pratiques qui y sont liées, de même que l'analyse de leur symbolique socioculturelle dans la présente contribution visent à montrer que les lieux de mémoire, au-delà de leurs aspects commémoratifs constituent des patrimoines, certes fragiles, mais incontournables dans la connaissance et la compréhension de l'univers culturel béninois.

\section{COMMUNES DE DASSA ET DE GLAZOUE}

Borgou

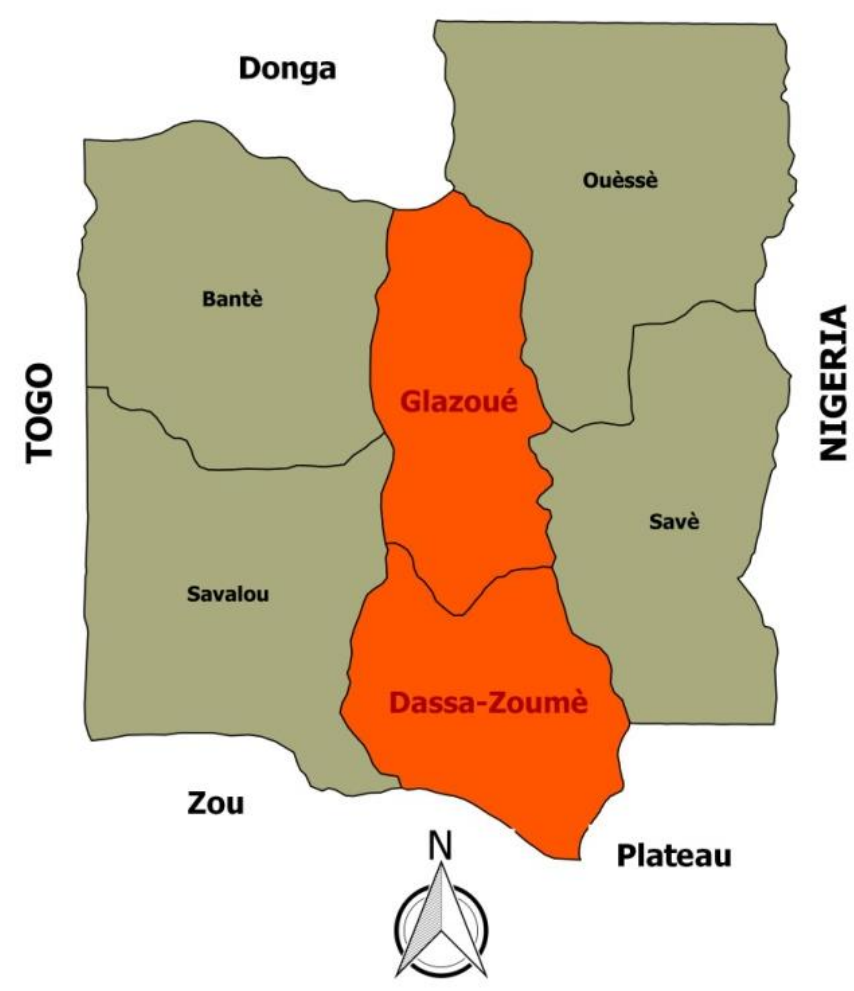

(C) Bokonon-Ganta Gildas 2015

Figure 1 : Carte du découpage administratif du département des Collines en communes.

\section{Les lieux de mémoire comme monuments de stimulation de la mémoire collective}

Une analyse du concept de "lieux de mémoire " nécessite de se pencher un tant soit peu sur le concept non moins important de la mémoire collective. Le concept de mémoire dans sa polysémie nous intéresse dans ce contexte en termes de lien entre le passé et le présent, comme le précise Lavabre (2007, p. 147) « la mémoire est tout à la fois trace et évocation du passé, effet du passé et effet du présent, interaction entre souvenirs de l'expérience et politiques de la mémoire ". La mobilisation des souvenirs et des expériences passées pour des manifestations présentes au sein d'une communauté peut se référer à l'idée de mémoire collective et nous pousse à nous interroger sur les processus de transformation de la multiplicité des mémoires individuelles à l'unicité des mémoires collectives. Le concept de mémoire collective prend son essor à travers les travaux considérés comme fondateurs dePierre Nora (1978) qui présente la mémoire collective comme ce qui reste du passé dans 
le vécu des groupes ou ce que ces groupes font de leur passé. II transparaît par conséquent que le groupe manifeste la volonté de célébrer les traces de ce passé collectif à travers des actions dans le présent. Ces célébrations se font généralement en associant des espaces, des lieux choisis sur des critères variés qui peuvent symboliser le lien avec un souvenir, refléter l'importance du lieu dans l'espace communautaire, etc. Ces espaces portent en général dans la mémoire commune du groupe, une force vitale qui a besoin d'être revitalisée continuellement. La présence des lieux de mémoire dans les espaces socioculturels béninois sont des marqueurs importants pour la compréhension de l'organisation spatio-culturelle. Chez les Idaatcha, la présence des autels des ancêtres dans les concessions familiales, au-delà des aspects rituels et religieux, sont des objets de mobilisation de la mémoire lignagère. Les marqueurs de la mémoire collective jouent leur rôle d'unificateur au sein des groupes les plus restreints comme la sphère familiale, mais également au sein d'ensembles plus larges comme l'aire culturelle, la nation, les communautés religieuses, etc.

Les lieux de mémoire sont par conséquent comme l'explique Sinou (2013, pp.184-185) à considérer comme des

[...]monuments au sens littéral du terme, c'est-à-dire des artefacts destinés à stimuler la mémoire, particulièrement utiles dans une société de tradition orale, où de surcroit les constructions édifiées en matériaux précaires et qui s'effacent avec le temps, ne sauraient suffire pour évoquer le passé. [...] Si temples, autels, couvents, arbres assurent pleinement leur fonction de monument et sacralisent l'espace, ils ne peuvent être considérés à priori comme des monuments historiques au sens occidental, c'està-dire comme des objets dotés de valeurs historiques et esthétiques définies à postériori (souvent plusieurs siècles après leur construction) par un corps de spécialistes usant de discours scientifiques pour les désigner comme tels.

La valeur patrimoniale des lieux de mémoire est de ce fait essentiellement liée à l'immatérialité des expressions mémorielles. Ces lieux, bien qu'étant des espaces physiques, n'ont d'importance spécifique qu'à travers les souvenirs, les commémorations, voire des esprits qui y sont attachés. En pays idaatcha chez les Oman-Jagou ${ }^{2}$ la pérennisation du souvenir, le renouvellement périodique de la commémoration est une réalité ancrée dans les pratiques socioculturelles. Les lieux de mémoire foisonnent sur le territoire d'Igbo Idaatcha et leur notoriété varie d'un lieu à un autre. Au nombre de ces lieux de mémoire qui servent à revitaliser la mémoire collective de la communauté des OmanJagou, Ègbakokou et Yaka font partie des plus importants et des plus connus.

\section{1 Ėgbakokou, un lieu de mémoire dans l'historiographie des Oman-Jagou : siège du pouvoir royal}

Le peuplement du royaume d'Igbo Idaatcha ne fait pas l'objet de publications scientifiques denses, toutefois, certaines études y ont été consacrées. Anignikin (2004) et Morel (1974) s'accordent à présenter le peuplement du royaume d'Igbo Idaatcha en plusieurs vagues migratoires, même si la chronologie des différentes vagues de migration ne fait pas l'unanimité. Anignikin (2004) défend une installation qui serait antérieure au $\mathrm{X}^{\mathrm{e}}$ siècle. Les Oman-Jagou seraient la dernière vague de migration. Les enquêtes de terrain ${ }^{3}$ confirment que les Oman-Jagou venus d'Ègba dans l'actuel Nigéria se seraient installés en dernière vague après les Idjéhoun et les Mamahoun. La réunification des différentes vagues pour fonder le royaume d'Igbo Idaatcha se serait effectuée entre le XVII et le XVIII ${ }^{\mathrm{e}}$ siècle (Anignikin, 2004, Morel 1974). Cette dernière vague de migration aurait été la plus importante en 
raison du nombre de migrants et a eu par la suite une grande influence dans l'organisation du territoire.

L'institution de la royauté à travers la réunification des quarante et une collines et par ricochet des territoires y afférents par les Oman-Jagou a permis à ces derniers d'asseoir leur hégémonie. Le pays idaatcha compte quarante et une collines, abritant des villages ayant à leur tête un Chef. L'ensemble des Chefs constituent l'Assemblée consultée par le Jagou (roi) pour les grandes décisions. Le palais royal "Odi » est installé au quartier Ègbakokou qui signifie littéralement en langue localeidaatcha « Ègba n'est pas mort ». Le toponyme Ègbakokou est assez expressif en termes d'enjeux mémoriels symbolisant la volonté collective des Oman-Jagou de pérenniser le souvenir de leur lieu de provenance Ègba qui se situe dans l'actuel Nigéria à leur emplacement actuel au Bénin. La dénomination Ègbakokou témoigne de la volonté manifeste de maintenir le lien entre le passé et le présent. Ègbakokou est par conséquent une représentation vivante de la mémoire d’Ègba, origine des Oman-Jagou sur leur territoire d'installation Igbo Idaatcha au Bénin actuel.

Odi, le site du palais royal, est juxtaposé à une grande place également dénommée Ègbakokou. Elle est marquée par la présence de représentations sacrées telles que :

- «Idi iwèoyé » qui signifie « lieu de purification des dignitaires ». Ce marqueur culturel de forme circulaire représente l'emplacement où le rituel d'intronisation du roi et de certains dignitaires se déroule. Dans le cadre de l'intronisation du roi, ce dernier est mis en retraite dans une case située à Issalou (quartier voisin d'Ègbakokou), la veille de l'événement. Le jour de l'intronisation, il est conduit par les sages à " Idi iwèoyé " pour le rituel de purification qui le mènera au palais. Assis dans un hamac, il est porté vers le palais via un sentier qui contourne les trois sillons sacrés.

- " "Oossa » est un marqueur cultuel du dignitaire qui assume le rôle de père de la communauté Oman-Jagou. II porte le nom de son titre et fait partie des symboles sacrés de la cour royale.

- les trois sillons sacrés ne portent aucune dénomination particulière. Ils auraient été aménagés pour commémorer la mémoire des trois épouses de N'kodéjou Arêmou, huitième Jagou d'Igbo Idaatcha, qui aurait été atteint de démence passagère et qui aurait assassiné ses trois épouses, avant de se suicider (Adjiba, 2006). Le sang des reines assassinées se serait écoulé en trois lignes, incitant les sages à tracer ces trois sillons pour rappeler ce tragique événement à la mémoire collective, afin que toute la communauté y soit sensibilisée. Les sillons sont entretenus par les Mamahoun à l'insu de tous et font partie du paysage spirituel de la cour royale. Ils sont inclus dans le parcours d'intronisation des rois et dans les rites funéraires. Il est formellement interdit de les enjamber.

Les illustrations ci-après montrent le marquage sommaire de ces sites sacrés qui singularisent l'espace d’Ègbakokou, qui depuis plusieurs siècles demeure le siège de la royauté dans son versant exécutif en pays idaatcha. 


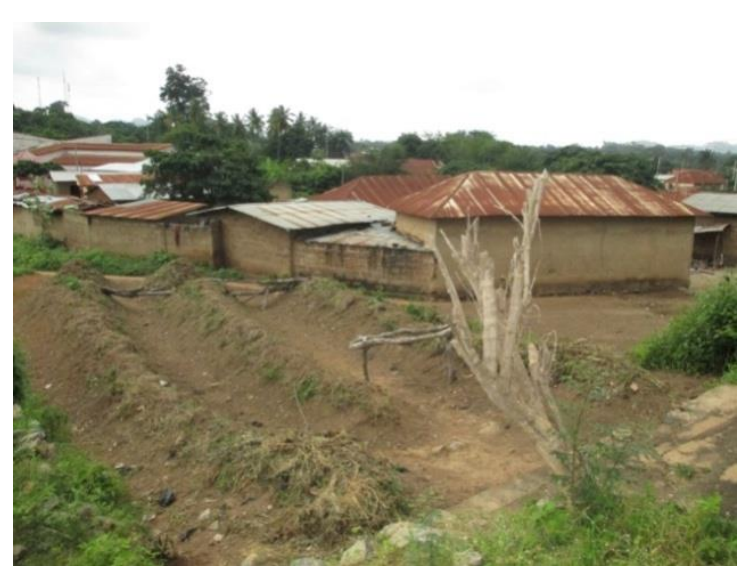

Figure 2 : Les trois sillons sacrés (C) B. Agbaka 2014

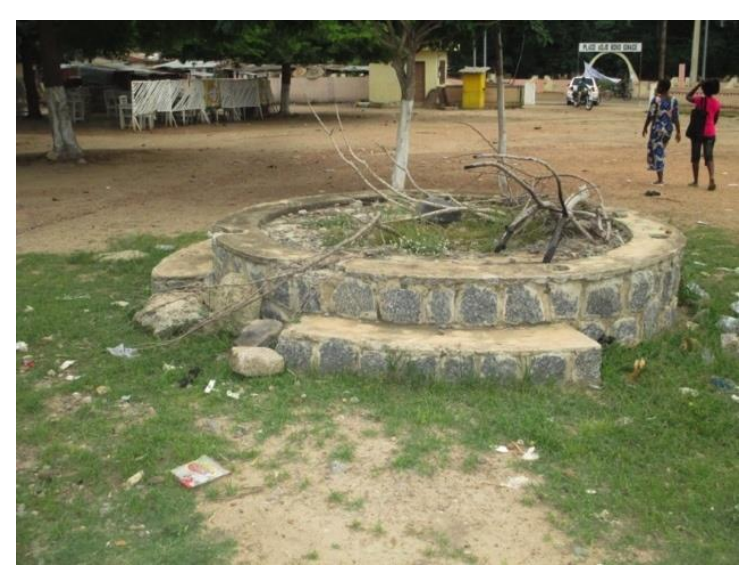

Figure $4:$ " Idiwè Oyé », lieu où sont pratiqués les rituels de purification pour I'intronisation du roi ou des dignitaires (C) B.Agbaka 2014

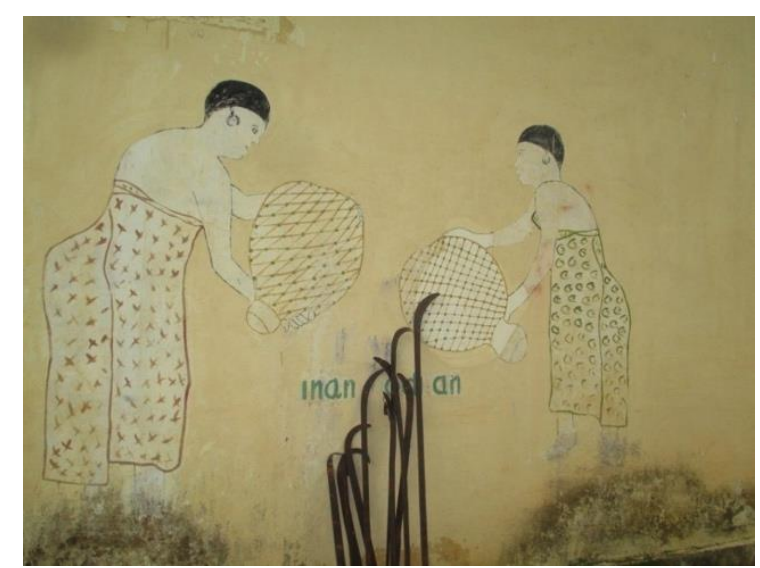

Figure 3 : Lieu de rituel des reines (C) B. Agbaka 2014

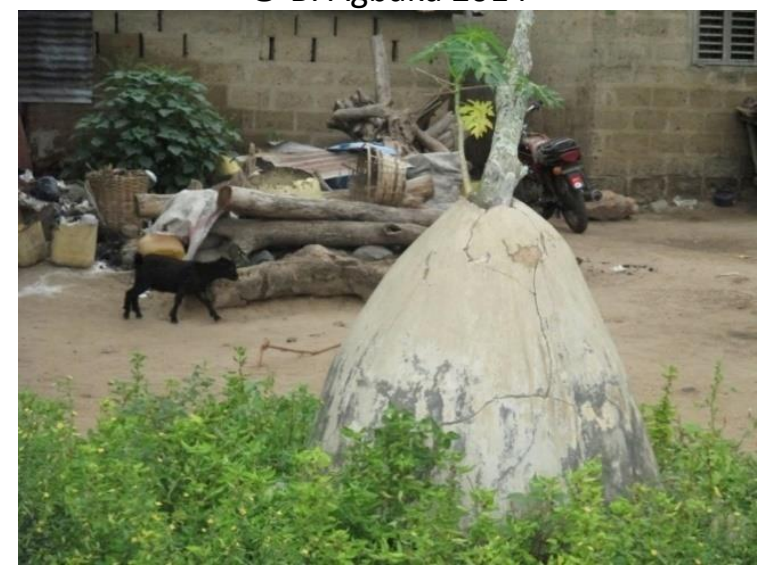

Figure 5 : site sacré du « Oossa »,

(c) B. Agbaka 2014

\subsection{Yaka, siège du pouvoir religieux : un sanctuaire entre passé et présent}

À proximité du palais royal d'Ègbakokou se dresse Yaka, le sanctuaire installé sur la colline Okéègnité. La signification de Yaka dériverait de l'allégorie "Yé a ka ti l'oké ». Les Oman-Jagou se comparent dans leurs louanges panégyriques à des feuilles qui tombent périodiquement de l'arbre. En tombant de l'arbre, la feuille aurait atterri sur la colline concernée. La signification de ce toponyme peut être considérée comme un début de compréhension du culte des ancêtres rendu sur la colline.

En effet, Yaka symbolise le pouvoir religieux qui dans l'organisation structurelle du royaume est séparé du pouvoir royal exécutif. L'espace de Yaka est sous la responsabilité du Obaloké4qui dirige toutes les cérémonies et tous les rituels d'envergure communautaire. Il incarne le pouvoir religieux sur un territoire où la spiritualité occupe une place capitale dans la vie quotidienne des populations.Yaka est l'altérité d'Ègbakokou qui est l'espace de prédilection du Jagou.

La symbolique de Yaka en tant que lieu de mémoire chez les Oman-Jagou a été construite sur de multiples objets de commémoration. II y a, en premier lieu, la symbolique religieuse qui reste prédominante et qui est portée par les autels de divinités et les autels de divinisation des ancêtres qui y sont installés. On y trouve également des vestiges de la résistance à l'esclavage dont témoignent les grottes refuges. En effet, ce territoire perché sur les hauteurs a servi de site refuge durant les périodes 
de guerres d'invasion menées par les royaumes voisins en vue de constituer des groupes de prisonniers destinés à servir d'esclaves.

\subsubsection{Yaka, entre symbolique mémorielle et pratiques religieuses}

\subsubsection{Le chemin vers Yaka, un parcours singulier}

Le chemin vers Yaka est jalonné de symboles et d'autels. Sur le sentier sinueux et abrupt qui mène vers le sanctuaire se trouvent deux reposoirs de dignitaires. Ce sont des blocs de pierre choisis pour servir de siège d'escale à ces dignitaires durant leur montée vers Yaka ou leur descente. Il est formellement interdit à toute autre personne de s'y asseoir. Le premier reposoir est celui d'Obaloké. Ce haut dignitaire, considéré comme le chef spirituel suprême est choisi par Ifâ (oracles) et intronisé à Yaka sur la colline. Après son intronisation, il doit y loger toute sa vie durant, sans pouvoir redescendre de la colline de son vivant. Seule sa dépouille mortelle est descendue après son décès pour être inhumée à Igbo $N^{\prime}$ la ${ }^{5}$. Obaloké est le grand chef coutumier qui préside toutes les cérémonies religieuses importantes de la communauté Oman-Jagou. La vie recluse que lui impose son statut de figure emblématique du pouvoir religieux est l'une des grandes difficultés liées à ce titre. Le second siège d'escale est réservé à Oossa. Ce dignitaire est considéré comme le père des Oman-Jagou, car il est responsable de la cohésion de la communauté et assure, le cas échéant, l'intérim du Jagou.

\subsubsection{La structuration de l'espace à Yaka: entre célébrations mémorielles et manifestations rituelles}

Yaka est un espace où s'entremêlent différentes actions de commémoration et des pratiques rituelles. Ce lieu de mémoire abrite plusieurs autels des ancêtres et de divinités, de même que des grottes ayant servi d'abris sous roche. Plusieurs symboles y sont érigés pour commémorer différents événements communautaires, mais d'autres représentations sont essentiellement liées à des pratiques magicoreligieuses. Ces symboles mémoriels ou religieux impliquent des manifestations dont l'envergure varie des plus ostentatoires au plus discrètes.

\section{- Les rites funéraires à Yaka}

Le déroulement des rites funéraires dans la communauté Oman-Jagou en pays idaatcha est une cérémonie bien organisée suivant plusieurs rituels. Le décès d'un Oman-Jagou participant déjà à la grande cérémonie rituelle annuelle de la réfection des cases ancestrales de Yaka dénommée " Iruwèrè » dont nous parlerons dans les lignes suivantes, induit entre toute autre manifestation, des rituels effectués à Yaka. Le rituel funéraire consiste à porter la dépouille du défunt à Yaka. Le cortège funèbre est accueilli dans un espace réservé à cet effet à l'entrée du sanctuaire. II s'en suit des rituels effectués sur l'autel dénommé "Anantchi ». La dépouille est installée dans une chambre funéraire pour la suite des rituels. Les défunts qui n'auraient pas été inhumés suivant les rites traditionnels de Yaka doivent faire l'objet de la cérémonie du « Igbèwolé ", c'est-à-dire le retour au bercail pour espérer que leurs esprits reposent auprès des ancêtres. À la fin des rites funéraires à Yaka, la dépouille est descendue pour être inhumée à "Igbo N'la », la grande forêt qui fait office de cimetière des OmanJagou. 


\section{- La case d'Ogoudou ou la célébration du roi-dieu à Yaka}

Ogoudou aurait été le plus puissant des Jagou. D’après la reconstitution de la royauté des Oman-Jagou effectuée par Palau Marti (1957) et Adjiba (2006), il aurait régné en quatrième position. Son règne aurait été le plus long, de 1538 à1572 (Adjiba, 2006). La divinisation de Jagou Ogoudou repose sur une légende qui raconte quevers la fin de son règne, en raison de sa sénilité, il ne pouvait plus assumer correctement les attributions royales, et aurait été isolé à Yaka dans une case. II se serait échappé de celle-ci sous la forme d'un serpent humanoïde ayant la tête du roi Ogoudou mais un corps de serpent. L'une des filles du roi, qui avait pour habitude de lui apporter son repas sur la colline, aurait été témoin de cette mutation extraordinaire et l'aurait rapportée à la communauté. Cette disparition mystérieuse de Jagou Ogoudou serait à l'origine de sa représentation, jusqu'à nos jours, sous la forme d'un serpent ayant une tête humaine. Dès lors, Jagou Ogoudou a été divinisé, et sa case d'isolement est devenue non seulement un lieu de mémoire à Yaka, mais également un lieu sacré où se perpétuent les rituels les plus importants de la communauté Oman-Jagou. Ogoudou est l'objet d'un culte et est imploré et invoqué à plusieurs occasions.

La réfection annuelle de la case d'Ogoudou fait l'objet d'une grande célébration à travers la cérémonie du « IruWèrè » qui signifie le port des pailles.

\section{- Le Iru Wèrè ou la réfection des cases ancestrales de Yaka}

Le " Iru wèrè " est une cérémonie traditionnelle très importante au pays idaatcha en général et en particulier pour les Oman-Jagou. II consiste à réfectionner tous les ans les toits des cases ancestrales, en particulier celle de JagouOgoudou qui sert aux rituels les plus sacrés de la communauté. Le jour du déroulement de la cérémonie est fixé par les dignitaires coutumiers et se tient généralement un jour du marché "Adjarou " ". II n'y a donc pas de date fixe, mais la cérémonie se déroule en saison sèche et souvent en février oumars. Une fois la date annoncée par les dignitaires coutumiers, les hommes apprêtent les pailles qu'ils disposent par lot. Après la phase de séchage des pailles, tous les OmanJagou orphelins de père, vivant à Dassa et ceux de la diaspora, venus pour la circonstance se rassemblent en deux grands groupes, les hommes d'un côté et les femmes de l'autre. Au moyen d'émissaires, ils coordonnent le départ qui se fait en file indienne, les femmes précédant les hommes en scandant le cri d’appel « HikéHikééé Hi ». Ce rituel du « Iruwèrè » est accompli exclusivement par les Oman-Jagou de sang, les épouses ne sont pas concernées.

Les filles ne reçoivent pas en héritage le bâton de cérémonie de leur père, seuls les garçons peuvent en hériter après le décès de ce dernier. Ils ne sont pas autorisés à prendre part aux rituels du vivant de leur père. Les hommes marchent donc avec leur bâton de cérémonie. La file qui semble interminable s'ébranle vers Éti Ékpo ${ }^{7}$, à Igbo Érémou ${ }^{8}$ ou à Kogbêrê ${ }^{9}$ suivant la disponibilité du chiendent pour le port des lots de paille. Chaque participant porte un lot de paille sur sa tête et la file remonte à Yaka pour les déposer.

Chaque orphelin de père dispose donc de son bâton de pèlerinage, " okpairu ». Ce bâton remis symboliquement après le décès du père vise à rappeler aux héritiers qu'ils héritent des charges de leurs parents. Le processus de transfert de responsabilité se fait normalement durant les rituels funéraires à Yaka pendant lesquels, le bâton « okpalru » ayant appartenu au père est donné en héritage au garçon aîné. Ce passage de bâton permet d'expliquer aux enfants venus enterrer leur père qu'ils 
doivent prendre la relève et que leur responsabilité est engagée devant toute la communauté. Ce rituel peut se rattraper si les enfants n'ont pas pu pour diverses raisons procéder aux funérailles suivant les rites traditionnels. Une fois le " okpairu » reçu des mains du chef coutumier, les enfants peuvent participer à la cérémonie d' "Iruwèrè ", suivante. Les filles participent à la cérémonie de transmission du bâton de cérémonie sans en hériter.

Durant le port des lots de paille, la très longue file constituée par les participants ne peut être traversée sous aucun prétexte. Le cortège s'ébranle, chacun portant son lot de paille sur la tête et marchant vers Yaka où la paille sera entassée, en attendant la réfection des cases " llé ti toché ". La paille est portée sur la tête à l'aide de feuilles de bananiers enroulées et attachées par des cordes fabriquées avec des ficelles prélevées sur le bananier. À la suite du "Iruwèrè ", les jeunes désignés au préalable procèdent à la réfection des toits sous l'œil vigilant des aînés.

En guise de contribution, les femmes sont chargées quelques semaines avant la cérémonie de " llé ti toché » de la responsabilité de nourrir ceux qui vont travailler à recouvrir les toits. Le procédé du choix des femmes devant assumer la restauration s'appelle "Assériran », qui pourrait se traduire par " commande de mets ». Les dignitaires prennent quelques ingrédients du mets à commander et les apportent aux différentes personnes choisies. C'est ainsi que lorsqu'une personne reçoit quelques grains de maïs et qu'on lui dit « Aran è l'assé » (" tu as été choisie pour la restauration »), elle comprend qu' elle doit cuisiner un mets à base de maïs (akassa, pâte de maïs, etc.) pour la cérémonie. La stratégie du "Assériran " est un système traditionnel d'entraide pour mettre en œuvre efficacement les événements concernant toute la communauté. En réalité, la personne qui reçoit la mission du « Assériran » ne s'en charge pas seule, elle se réfère à toute sa famille et à ses proches pour obtenir leur contribution.

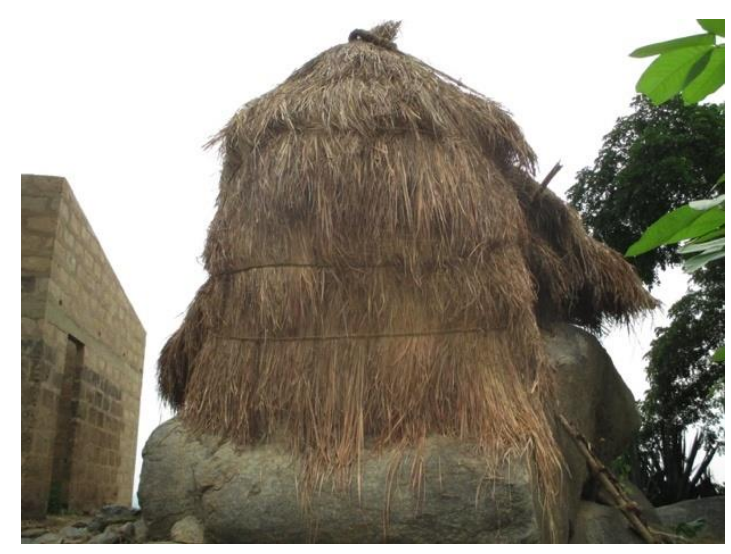

Figure 6 : case rituelle de JagouOgoudou

(C) B. Agbaka 2014

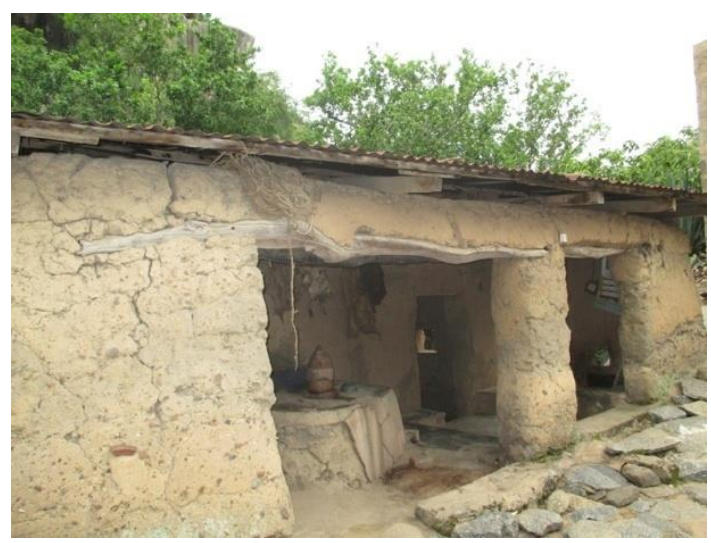

Figure 7 : case rituelle dédiée aux ancêtres

(C) B. Agbaka 2014

- Ikodoun, la fête des prémices à Yaka

Yaka abrite également les manifestations d'Ikodoun, la fête des prémices chez les Oman-Jagou. La fête d'Ikodoun est précédée du rituel " d'odjarirè ", qui consiste à prélever au marché auprès de toutes les vendeuses et tous les vendeurs, les vivres exposés. Ceux qui vendent des articles assez couteux sont invités à faire un don symbolique en espèce. En effet, cette fête communautaire permet à la communauté de remercier les ancêtres pour l'année écoulée et de présenter les produits de la 
nouvelle récolte. II est formellement interdit aux membres de la communauté de goûter au maïs fraichement récolté avant la cérémonie des prémices. Ikodoun se déroule environ trois mois après la cérémonie du "Iruwèrè ". Elle permet de rendre le culte dû aux ancêtres et de solliciter leurs bénédictions pour l'année nouvelle. À cette occasion, les problèmes collectifs relatifs à la vie communautaire sont débattus et des solutions sont envisagées. Ikodoun se fête en deux parties : une partie rituelle et une partie festive qui se manifeste avec l'accueil des invités. La partie rituelle consiste à offrir sur l'autel des ancêtres, situé devant la case servant aux rituels funéraires, la bière traditionnelle à base de maïs et de mil dénommée "Tchakparo ".

La bière locale sert pour les libations et un bélier est offert en sacrifice. Ce rituel aux ancêtres" Iwouré » est présidé par les " Inan ", c'est-à-dire " mères " qui sont chargées de mener toutes les prières en présence de tous les autres dignitaires. La fin du « Iwouré » fait place aux festivités.

À Yaka, lors des cérémonies rituelles, les dignitaires, chacun disposant de son siège, s'assoiventsuivant un ordre bien déterminé. Il est formellement interdit à tout dignitaire de se tromper de siège, sous peine de sacrifier à un rituel de lavement de l'offense infligée au dignitaire dont le contrevenant a pris le siège en offrant un bélier. La case des dignitaires qui fait office de parlement pour discuter des grandes décisions liées à la vie de la société et ayant un aspect lié aux cultes traditionnels ou nécessitant l'intervention des ancêtres abrite des sièges bien fixés pour chaque dignitaire participant à l’assemblée.

La disposition des sièges à l'intérieur de l'espace des dignitaires à Yaka se présente comme suit : 
Siège Obaloké (1) chef coutumier suprême

\section{Entrée 3}

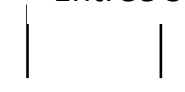

Réceptacle des

Autel des ancêtres

offrandes

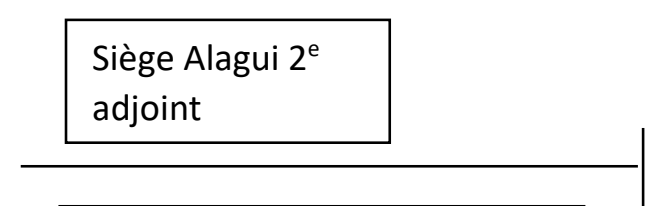

Siège Oossa (1) Père du peuple

Sièges

Odjadian

et Owo

Aman

(adjoints
Entrée 2

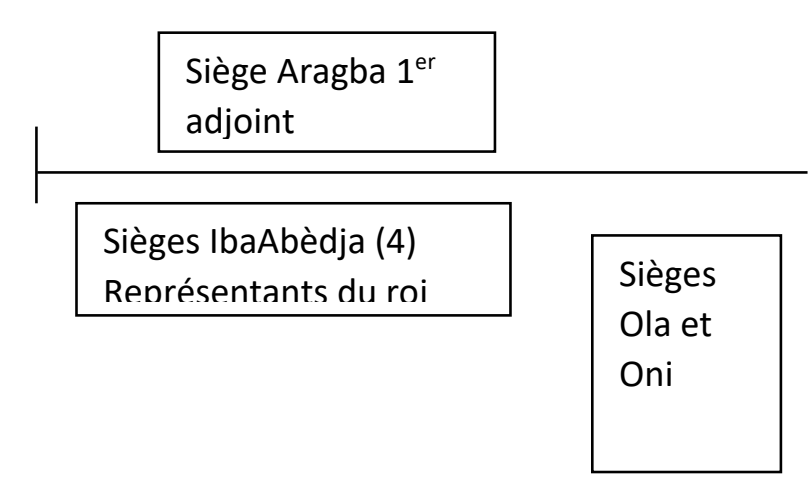

\section{Oga (1) \\ Organisateur}

Alourin (1)

Ibalshouokp

o (4)

gardiens des

bâtons de

cérémonie

Entrée

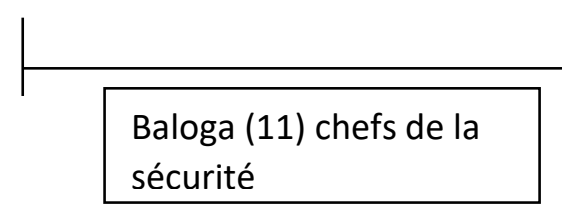

sécurité
Alaritan (23)

chefs de collectivités et de culte familial

Figure 2 : Disposition des sièges dans l'espace des dignitaires à Yaka 


\section{- Ifo Oyoro ou les grottes refuges de la reine Oyoro}

Oyoro, fille du premier roi JagouOlofin, aurait régné en deuxième Jagou à la suite de son père de 1526 à1530 (Adjiba, 2006). La reine Oyoro se serait installée sur la Colline de Yaka dans le but de protéger son peuple des invasions guerrières. Elle aurait installé son palais dans la grotte dénommée « IfoOyoro ", c'est-à-dire la " grotte d'Oyoro ", et son peuple autour de la colline appelée Kpêmbêlè. Cette grotte comporte encore de nos jours des objets qui auraient appartenu à la reine d'après les conservateurs locaux du site. Toutefois, la véracité scientifique des ces reliques ne peut être attestée que par des fouilles archéologiques.

Yaka abrite également l'autel des divinités Ogou, dieu du fer et de la guerre, Nana Buku, divinité protectrice des Oman-Jagou, plusieurs grottes servant d'autels à d'autres divinités, un cimetière de dignitaires ainsi que des habitations perchées sur les collines. Celles-ci sont habitées en partie par certains "Oman-Ogou », adeptes de la divinité du fer et de la guerre. Cette expression désigne également les chasseurs qui, de par leurs pratiques, sont adeptes de cette divinité protectrice de leurs activités.

\subsubsection{Les réfections et modifications de biens patrimoniaux à Yaka, un objet de discorde entre communautés et professionnels}

Le sanctuaire de Yaka fait partie des sites de la Route de l'esclave au Bénin. Étant donné que le gouvernement béninois travaille à l'inscription de la Route de l'esclave sur la Liste du patrimoine mondial, cela induit le respect des exigences en matière d'inscription dans la conservation du site. Toutefois, ces exigences ne correspondent pas souvent à la vision des communautés détentrices qui interviennent librement sur le site sans nécessairement faire recours à l'accord des professionnels de la Direction du patrimoine culturel. Les dissensions entre les dignitaires de Yaka et les professionnels sont essentiellement liées aux différentes réfections des cases rituelles qui font l'objet de reconstruction en briques de ciment et de recouvrement de la toiture avec des tôles ondulées, à l'exception de la case de Jagou Ogoudou dont la réfection maintient son architecture traditionnelle. Par ailleurs, de nouvelles constructions sont érigées sur le site sans l'accord de la Direction du patrimoine culturel, et ces nouveaux bâtiments posent un sérieux problème de préservation de l'authenticité du site. En effet, au regard des normes professionnelles de conservation du patrimoine, il est inadmissible qu'un site comme Yaka, qui à l'origine ne comportait que des matériaux traditionnels de construction, arbore désormais des constructions qui ne font plus usage ni du banco, ni de la paille.

Les difficultés de conservation du paysage culturel de Yakapose le problème récurrent de la conservation des sites africains en général et ceux béninois en particulier suivant les normes internationales, fortement occidentalisées. Au-delà d'un désir ardent des communautés de se moderniser en faisant usage de matériaux en vogue, il existe un sérieux problème de disponibilité des matériaux de construction traditionnels et de la survie des savoir-faire liés à ce type de construction. Les pailles servant à la reconstruction des cases à Yaka sont de moins en moins accessibles en raison de la pression immobilière qui amenuise au fil des jours la superficie des forêts sacrées constituant les réserves de pailles. La rareté des matériaux traditionnels incite les populations à adopter des matériaux estimés plus pérennes. Les corps de métier liés à la construction du bâti traditionnel sont en voie de disparition parce qu'ils ne font plus vivre les praticiens. Le dialogue devient dès lors souvent compliqué 
entre les acteurs traditionnels de la conservation de Yaka et les experts qui veulent maintenir les sites dans toute leur authenticité.

\section{Lieux de mémoire et authenticité au Bénin : quelle réflexion pour une meilleure collaboration entre professionnels et communautés}

Les difficultés de collaboration entre communautés et professionnels autour du patrimoine en général et des lieux de mémoire en particulier peuvent avoir plusieurs causes ; les plus importantes étant les débats sur la question de l'authenticité en Afrique, les difficultés d'investissement des pouvoirs publics dans la restauration, ainsi que la conservation et la valorisation du patrimoine. La problématique de I'authenticité sur les lieux de mémoire au Bénin est une réelle écharde dans les mains des professionnels qui dans leurs efforts pour se conformer aux normes internationales, se voient souvent confronter à la réticence des communautés locales qui ne partagent généralement pas leurs exigences.

L'un des exemples les plus éloquents est le parcours de la Route de l'esclave dont les tribulations quant à son inscription au patrimoine mondial incitent à réfléchir sur la place de l'authenticité sur les lieux de mémoire. Cette question fait débat au sein des professionnels africains.

Munjeri (2003) aborde l'inadéquation des critères de l'authenticité tels que promus par les organisations internationales comme I'UNESCO, I'ICOMOS, etc. avec les réalités africaines. Ce professionnel du patrimoine africain explique que l'authenticité s'attache essentiellement aux aspects physiques du bien culturel, ce qui ne correspond souvent pas à la vision des communautés africaines. En matière d'authenticité des matériaux, qui se focalise sur l'essence physique du bien culturel, Munjeri (2003) rappelle lors de la réunion sur " l'Authenticité et l'Intégrité dans un Contexte Africain ", organisée par le comité du patrimoine mondial en 2000 au monument national du Grand Zimbabwe, que dans les sociétés à cultures vivantes, l'authenticité d'un vestige est plus liée à ce qu'il représentait qu'à sa forme physique. En matière d'authenticité de l'exécution, la fonction semble avoir plus d'importance que les considérations esthétiques. L'authenticité de la conception dans les cultures vivantes accorde plus d'importance au message qu'aux intentions de l'architecte, de l'artiste ou de l'ingénieur. II conclut son argumentaire par le fait que la notion d'authenticité en matière de patrimoine culturel est une notion très relative qu'il convient de fortement nuancer suivant les contextes socioculturels.

Les lieux de mémoire cumulant le mémoriel et le topographique n'échappent donc pas aux spéculations sur l'authenticité. La question qui nous semble importante de se poser et dont la réponse serait un début de solution aux incompréhensions liées à la gestion du patrimoine culturel au Bénin en général et des lieux de mémoire en particulier est : à qui revient-il de juger de l'authenticité d'un site? II nous semble que tant que les détenteurs d'un lieu de mémoire donné n'estiment pas son authenticité disparue, il revient aux experts de trouver les stratégies nécessaires pour faire comprendre aux populations concernées l'importance de maintenir la véracité de leur site tout en restant ouverts aux perceptions des communautés locales.La réelle survie de la mémoire collective et des lieux y afférents dépend de la réappropriation et de la flexibilité qu'il faut inclure dans la perception de l'authenticité, particulièrement dans des contextes comme ceux des populations africaines où l'essentiel du patrimoine est immatériel. 


\section{Conclusion}

Ègbakokou et Yaka sont des lieux de mémoire incontournables dans l'organisation spatio-temporelle et les pratiques socioculturelles des Idaatcha du Bénin. Ces deux lieux portent de nombreux marqueurs de la mémoire collective mais représentent également des symboles de pouvoir, car le pouvoir en pays idaatcha est partagé entre la royauté et la religion. Cette dualité est symbolisée par les lieux où siègent les deux figures du pouvoir : le Jagou et l'Obaloké. Le premier règne à Ègbakokou et le second à Yaka, mais les deux règnent dans la complémentarité pour conduire les destinées du royaume d'Igbo Idaatcha. Le maintien de la vitalité de ces lieux implique des manifestations qui peuvent être l'objet de désaccords avec les professionnels du patrimoine en matière de conservation et d'authenticité. Toutefois, la célébration continuelle de la mémoire étant capitale pour la survie de ces lieux, leur gestion doit s'appuyer essentiellement sur la contribution des communautés détentrices. La décentralisation administrative survenue au Bénin depuis environ deux décennies doit être un levier pour renforcer et favoriser le maintien de la collaboration qui existe entre système de gouvernance traditionnelle et administration décentralisée à travers les communes. La survie de plusieurs sites patrimoniaux en dépend.

${ }^{1}$ Igbo Idaatcha signifie forêt des Idaactha.

${ }_{2}$ Oman-Jagou signifie guerriers

${ }^{3}$ Enquêtes de terrain de juin à août 2014 et 2015 auprès de personnes ressources d'Igbo Idaatcha : le porte parole du roi, Oga, le Chef de la communauté Oman-Jagou et autres dignitaires.

${ }^{4}$ Roi des hauteurs

${ }^{5}$ Cimetière des Oman-Jagou

${ }^{6}$ Adjarou est en termes appropriés Odjaèrou (marché des esclaves), on y faisait le tri des esclaves avant de les conduire vers plusieurs destinations.

${ }^{7}$ Premier noyau historique du royaume d'Igbo Idaatcha.

${ }^{8}$ Forêt sacrée accueillant des rituels, son accès est interdit à tout profane.

${ }^{9}$ Kogbêrê est un autre lieu de mémoire à Igbo Idaatcha. On y commémore la disparition mystérieuse de Jagou Shagbona

\section{Références}

Adjiba, R.(2006).Les rois de Dassa, Cotonou, Les Presses de l'imprimerie Tundé.

Anignikin, C.S. (2004). Histoire des populations Idaatcha : à propos des manipulations du discours historique. DansAnnales de la Faculté des Lettres, Arts et Sciences Humaines. Vol. 9, 57-94.

Lavabre, M-C. (2007). Paradigmes de la mémoire. Dans Transcontinentales. Vol. 5, 139-147.

Morel, A. (1974). Un exemple d'urbanisation en Afrique occidentale : Dassa-Zoumè (Dahomey). Dans Cahier d'études africaines. Vol. 56, 727-748.

Munjeri, D. (2003). "Patrimoine immatériel en Afrique : s'agirait-il de beaucoup de bruit pour rien ? ", 14 Assemblée Générale et Symposium Scientifique de l'ICOMOS, 27-31 octobre.

Nora. P. (1984). 1997, Entre mémoire et histoire : la problématique des lieux. Dans Les lieux de mémoire, éd. P. Nora, Paris, Gallimard, 22-43.

Nora. P. (1978). La mémoire collective. Dans La nouvelle histoire, éd. R. Chartier, J. Le Goff, J. Revel, Paris, Retz . 
Palau-Marti, M. (1957). Notes sur les rois de Daşa (Dahomey, A.O.F). Dans Journal de la société des Africanistes. Vol 27(2), 197-209. 